\title{
Immature survival, fertility, and density dependence drive global population dynamics in a long-lived species
}

\author{
M. Genovart, ${ }^{1,2,4}$ D. Oro, ${ }^{1,2}$ and S. Tenan ${ }^{3}$ \\ ${ }^{1}$ CEAB (CSIC), Accés Cala Sant Francesc 14, 17300 Blanes, Catalonia, Spain \\ ${ }^{2}$ IMEDEA (CSIC-UIB), Miquel Marquès 21, 07190 Esporles, Spain \\ ${ }^{3}$ MUSE - Museo delle Scienze, Sezione Zoologia dei Vertebrati, Corso del Lavoro e della Scienza 3, 38122 Trento, Italy
}

\begin{abstract}
Disentangling the influence of demographic parameters and the role of density dependence on species' population dynamics is a challenge, especially when fractions of the population are unobservable. Additionally, due to the difficulty of gathering data at large spatial scales, most studies ignore the global dynamic of a species, which would integrate local heterogeneity dynamics and remove the noise of dispersal. We developed an integrated population model (IPM) at a global scale to disentangle the main demographic drivers of population dynamics in a long-lived species. We used $28 \mathrm{yr}$ of Audouin's Gull demographic data encompassing 69 local patches (comprising $90 \%$ of the world population). Importantly, we took into account the unobservable fraction of non-breeders and also assessed the strength of density dependence for this fraction of the population. As predicted by life histories of long-lived organisms, temporal random variation in survival was highest for immature individuals (1.326, 95\% credible interval [CRI] $1.290-1.940)$ and lowest for adults $(0.499,95 \%$ CRI $0.487-0.720$ ). Large temporal fluctuations in the probability of taking a reproductive sabbatical would partly explain the consistency in adult survival, with individuals most likely refraining from breeding when environmental conditions were harsh. Immature survival and fertility were the main drivers of population dynamics during the study period $\left(r^{2}=0.83,0.77-0.87\right.$ and $0.73,0.63-0.79$, respectively). We found strong evidence of density dependence, not only due to the number of breeders $\left(r^{2}=-0.34\right.$, -0.43 to -0.24$)$ but also due to individuals on sabbatical $\left(r^{2}=-0.18,-0.33\right.$ to -0.01$)$. From a conservation point of view, the species shows a $5 \%$ annual global decrease during the last 10 years, and we propose an update of its conservation status. Even though population dynamics of long-lived organisms are very sensitive to changes in adult survival, we show here that, in the absence of strong environmental perturbations affecting this vital rate, fluctuations in population density are mainly driven by variations in survival of immature individuals and fertility. Integrated models based on long-term monitoring at a global scale may enhance our ecological and evolutionary understanding of how demographic drivers influence population dynamics.
\end{abstract}

Key words: Audouin's Gull; colonial seabird; demographic drivers; density dependence; integrated population model; long-lived species; non-breeders; population growth rate; sabbatical; survival; unobservable states.

\section{INTRODUCTION}

Estimating demographic parameters and understanding their role in driving population dynamics are crucial from both a theoretical and applied perspective. Life history theory predicts and prospective analyses have shown that the contribution of the demographic rates to the population growth rate varies with species life history traits, and population dynamics of long-lived species are very sensitive to variations in adult survival (Stearns 1992, Caswell 2000, Sæther and Bakke 2000). However, fitness components that have a large influence on population growth may exhibit low temporal variation (Sæther and Bakke 2000, Gaillard and Yoccoz 2003, Morris and Doak 2004, Bjørkvoll et al. 2016) and this may effectively reduce their influence in population dynamics.

Important differences may also exist in demographic parameters within a species at spatial and temporal scales (Frederiksen et al. 2005, Grosbois et al. 2008, 2009, Genovart et al. 2018). Dispersal may play a critical role in many

Manuscript received 20 February 2018; revised 2 August 2018; accepted 22 August 2018. Corresponding Editor: John Sauer.

${ }^{4}$ E-mail: m.genovart@csic.es species' population dynamics (Stenseth and Lidicker 1992, Clobert et al. 2009). Due to the difficulty of gathering data at a global scale, most studies are local or include only a few local populations, ignoring global species dynamics. Demographic parameters and their influence on population dynamics may change among different fractions of the population (e.g., age, sex). Assessing the role of these fractions can be challenging if they are not easily observable or monitored. This unobservable fraction may include one or more age classes, and it is usually unobservable due to a specific behavior that makes individuals more cryptic or not visible to the eye of the researcher. For example, males in marine turtle populations do not reach the beach for breeding and therefore are not catchable (Crouse et al. 1987) while some animals undergo torpor and become unobservable (Ganzhorn and Schmid 1998). Also in many species (e.g., some birds and amphibians), there is a non-breeding fraction of the population that are difficult to study (Cam et al. 1998, Bailey et al. 2004, Muths et al. 2010). This unobservable, non-breeding fraction of the population may include sexually mature individuals that have not yet been recruited and those that are already recruited but skip breeding. For these species, population dynamic studies are usually based only on the reachable breeding fraction of the population. The 
prevalence and role of non-breeders in population dynamics are unknown (but see Pardo et al. 2013). Lee et al. (2017) recently highlighted the importance of including the nonbreeding segment of the population when assessing population growth rates.

Density dependence, defined as a positive (Allee effect) or negative effect of population size on population growth rate, can also play a major role in regulating population dynamics through resource competition (Hanski et al. 1996, Sibly and Hone 2002, Lande et al. 2003, Sæther et al. 2016). The importance of density dependence in population ecology and quantifying its role is often analytically challenging (Dennis et al. 2006, Freckleton et al. 2006, Knape 2008). Lebreton and Gimenez (2013) highlight the potential biases when assessing the strength of density dependence either on population dynamics or life history traits. Integrated population models (IPMs) are useful tools for inferring population growth and age-specific demographic rates. IPMs allow for joint analysis of demographic data originating from different sources of information (Schaub and Abadi 2011, Tenan et al. 2012, 2016). These integrated models reduce parameter uncertainty, incorporate variances and covariances between different demographic parameters into model projections, account for sampling variance, and allow for the estimation of latent parameters and the strength of density dependence (Abadi et al. 2010, 2012, Schaub and Abadi 2011).

The Audouin's Gull Larus audouinii is a long-lived seabird that was critically endangered around the 1980s. Due to the establishment of one colony in 1981 and its exponential growth, the species has recently been downgraded to "least concern" (BirdLife International 2017). However, this colony is now collapsing, holding from more than $70 \%$ of the total world population in 2006 to only 3\% in 2017 (Authors, unpublished data). At the same time, some other new patches have been recently colonized (Payo-Payo et al. 2017) and the actual global conservation status of the species is unknown, placing a spotlight on the conservation of this species yet again.

We developed an IPM to analyze an extremely rich database covering almost the entire breeding range $(90 \%$ of the total breeding population) of the Audouin's Gull, including annual breeding census information, fertility data, and capture-recapture data. Using this approach, our aims were to (1) disentangle the demographic drivers of population dynamics in a long-lived species at a global scale, (2) evaluate the importance of density dependence at each fraction of the population, including the unobservable non-breeders, and (3) assess the global conservation status for the species. These results will help to improve our understanding of the demographic parameters that drive population dynamics in long-lived species.

\section{Materials ANd Methods}

\section{Study species, study areas and data sets}

The Audouin's Gull is a long-lived colonial seabird with more than $90 \%$ of the global population actually breeding in the western Mediterranean (data available online). ${ }^{5}$ An

\footnotetext{
${ }^{5}$ http://www.iucnredlist.org/details/22694313/0
}

extensive long-term monitoring program has been carried out mainly at the larger colony (Punta de la Banya, Catalonia; $40^{\circ} 37^{\prime} \mathrm{N}, 00^{\circ} 35^{\prime} \mathrm{E}$ ), but also to a lesser extent at other breeding sites, including some recently colonized patches (Fernández-Chacón et al. 2013, Payo-Payo et al. 2017). From this monitoring, three data sets have been built: annual breeding population counts, individual encounter histories of capture-recapture data and fertility data (Fig. 1).

Breeding population counts.-Annual censuses of breeding pairs at western Mediterranean sites were made from 1988 to 2015 at most of the breeding colonies. Even if we could not guarantee that all colonies were registered annually, we are confident we covered the most important ones each year; we ended up monitoring 69 different sites throughout the study period.

Individual encounter histories.-During 1988-2015, a total of 27,290 individuals were captured and banded as fledglings using a darvic band with a unique alphanumeric code at the Punta de la Banya colony (Oro et al. 2010, Genovart et al. 2012). During the study period, resightings were made using spotting scopes from a distance all over the western Mediterranean with a total of 63,106 resights.

Fertility data.-Fertility data set includes the total number of fledglings for each year from 1988 to 2015. The number of fledglings at each colony was estimated by capture-recapture methods (Lincoln-Peterson method, Nichols 1992). For those colonies for which we did not know the number of fledglings, it was assumed to be the product of the number of breeding pairs in the colony by the mean fertility estimated for that year in all the colonies where number of fledglings was known.

\section{The integrated population model}

We implemented an integrated analysis of the three data sets (breeding population count data, individual capturereencounter histories, and fertility data). We used an IPM (Besbeas et al. 2002) to obtain consensual estimates of demographic rates and population sizes, where inference is based on a joint likelihood derived by the multiplication of the likelihoods for the data sets (Schaub and Abadi 2011).

We used a state-space model for population count data, where the observation process is conditional on a state process that describes changes in population size over time as a function of demographic rates through a population projection model. We used a female-based, pre-breeding, census model with nine stages defined by the combination of individual age and breeding status and based on previous studies on this species at its larger colony (Payo-Payo et al. 2016, Genovart et al. 2017). Specifically, we distinguished stages for one- and two-year-old individuals (juveniles and immatures that are not sexually mature), three stages for not-yet recruited individuals (three-, four-, and five-year-old or older individuals that have never bred), three stages for breeders (three-, four-, and five-year-old or older individuals), and another stage for sabbaticals (i.e., individuals that have bred at least once and skip reproduction). Changes in 

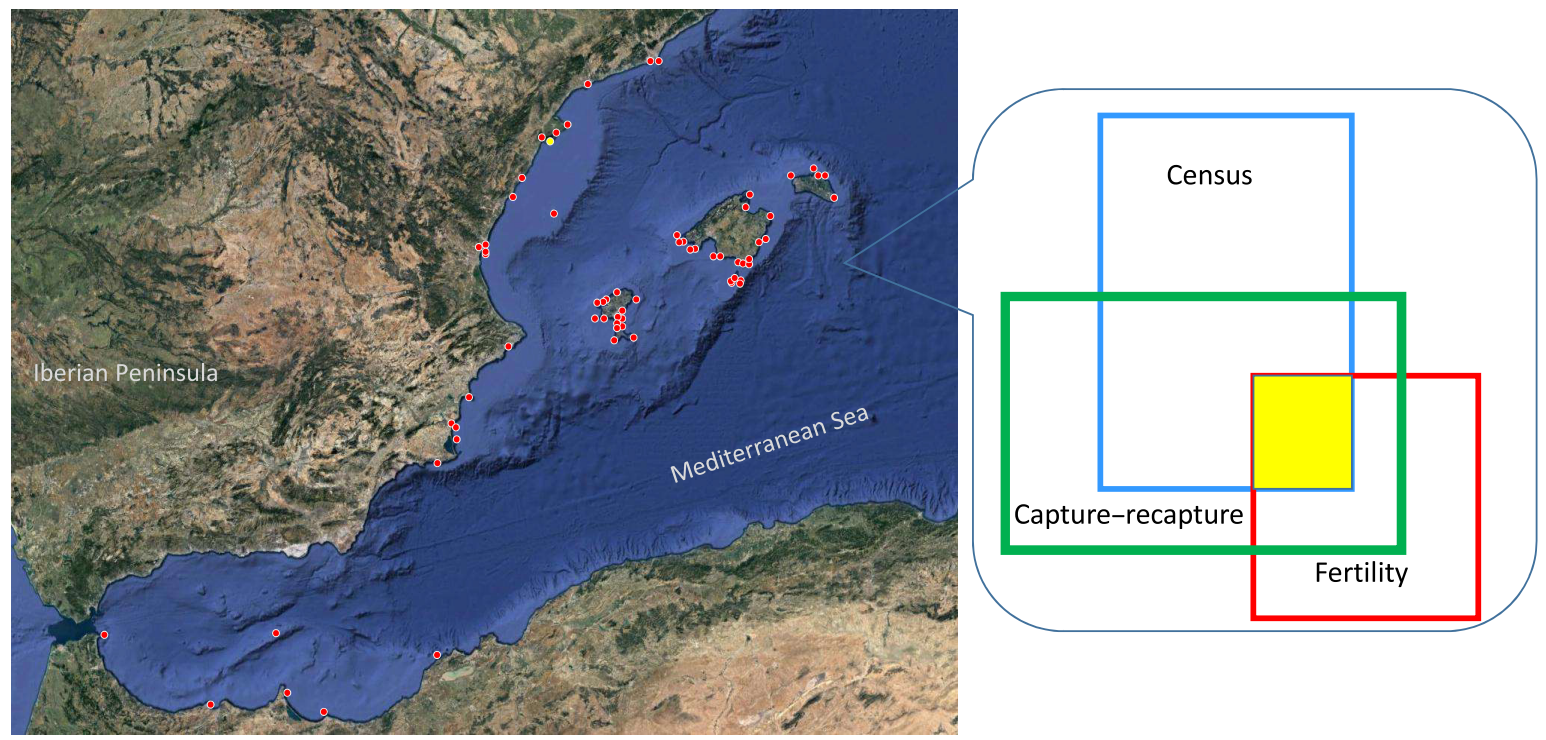

FIG. 1. Map of the study area comprising $90 \%$ of the global species distribution and 69 different breeding sites censused from 1988 to 2015. Census, fertility, and capture-recapture data were jointly analyzed in the integrated population model (IPM).

population sizes of age-stage classes between consecutive years were stochastically modeled using binomial and Poisson distributions (Appendix S1: Eqs. S1-S4, S6-S7, S9-S13, S15-S16, S18-S22). We assumed no immigration occurred in the study population because we are modelling most of the global population. We also formulated the state-space model with the assumption of an even sex ratio of fledglings (Genovart et al. 2003, see Appendix S1) and that individuals can start reproducing for the first time at three years of age (Oro and Pradel 2000). We defined age-specific probabilities for survival: $\phi_{a 12}$ for one- and two-year-old individuals, $\phi_{a 3}$ for three-year-old individuals, $\phi_{a 4}$ for four-year-old individuals, and $\phi_{a 5}$ for five-year-old or older individuals. We also specified age-specific probabilities of first reproduction at three years of age $\left(\alpha_{a 3}\right)$, four years of age $\left(\alpha_{a 4}\right)$, or older $\left(\alpha_{a 5}\right)$, and age-independent probability to skip reproduction (Sb) once recruited. In the observation model, we described the relationship between counts of breeding females at year $t\left(y_{t}\right)$ and the estimated colony size, assuming that the observation error was normally distributed on the log scale (Appendix S1: Eqs. S27-S28). The latter assumption implies that counts of breeding pairs are unbiased on average, that is, false-negative and false-positive observations cancel out on average (Kery and Schaub 2011). In addition, the small error rate in the breeding population surveys (3-4\%, Oro and Ruxton 2001) supports the assumption of negligible error in detection and that the state-space sub-model yields unbiased estimates of population size (Kery and Schaub 2011).

We modeled individual encounter histories with a Cormack-Jolly-Seber (CJS) model (CJS; Lebreton et al. 1992) based on previous studies on this species at its larger colony (Payo-Payo et al. 2016, Genovart et al. 2017). In the CJS, we considered age differences in survival and encounter probability, between the four above-mentioned classes for survival and three age classes for encounter probability (one-, two-, and three-year-old or older individuals), in addition to trap response, and random time variation in both parameters (Appendix S1: Eqs. S29-S42). We used a Poisson process to model data on reproductive success (Appendix S1: Eq. S43). The integrated population model was formulated to estimate the temporal random variation in fertility $(b)$, survival rates $(\phi)$, probability to skip reproduction $(\mathrm{Sb})$, and encounter probability $(p)$. We thus used a hierarchical formulation where annual estimates were assumed to originate from a random process with a common mean and a constant temporal variance (Appendix S1: Eqs. S31-S32, S44, S45). Probabilities of first reproduction $(\alpha)$ were modeled as time invariant using informative priors based on estimates derived for the same population and reported in Genovart et al. (2017) (Appendix S1: Eqs. S46-S48). Mean and 95\% credible interval $(95 \% \mathrm{CRI})$ values for all estimates were provided throughout the manuscript.

\section{Model implementation}

We fitted the integrated population model using a Bayesian formulation and the Markov chain Monte Carlo framework. Summaries of the posterior distribution were calculated from $3,000,000$ posterior samples (20 chains, thinning of 100 , and burn-in of 500,000 iterations). We assessed convergence using the $\hat{R}$ diagnostics (Brooks and Gelman 1998) that was $<1.01$ for all parameters. We refer to Appendix S1 and the supplementary model code in Data $\mathrm{S} 1$ for details about prior distributions. Models were implemented using the program JAGS (Plummer 2003) executed from R (R Core Team 2012) using rjags package (Plummer 2016).

\section{Effects of vital rates on population growth rate}

We compared temporal variation of vital rates with the variation in population growth rate to assess the differential influence of those rates on population dynamics. Specifically, we used correlation analysis that accounted for uncertainty in parameter estimates, where correlation coefficients $(r)$, their $95 \%$ credible interval $(95 \% \mathrm{CRI})$, and the 
probability that $r$ was positive or negative were derived using posterior samples of population growth rate and vital rates on the real scale in a post-hoc analysis (Tenan et al. 2017). In addition, the explicit separation of the process and sampling variation, within the integrated population model, ensured correlations to be free from possible bias related to the observation process; temporal variability of the state process is thus defined by the variability of the demographic parameters (Schaub et al. 2013).

\section{Density dependence}

We correlated estimates of population growth rate between year $t$ and $t+1$ with total population size and stage- or age-specific number of individuals in the population at year $t$, in order to assess the strength of density dependence and how the latter depends on population size of specific age-stage classes: number of 1-yr-old individuals, 2-yr-old individuals, 3-yr-old individuals, breeders, sabbaticals, not-yet recruited, and non-breeders (i.e., not-yet recruited and sabbaticals).

\section{RESULTS}

The estimated total population size reached its maximum of 34,109 (32,747-35,475 95\% CRI) individuals in 2007, followed by a marked decrease that led to a halved population size of 17,367 individuals $(16,652-18,138)$ in 2013 (Fig. 2; Table S1 in Appendix S2). The geometric mean of population growth rate $\lambda$ for the 28-yr study period (1988-2015) was 1.002 (1.000-1.007, Fig. 3a), and 0.949 (0.929-0.963) for the last 10 years.

The estimated number of breeding pairs varied from 6,834 $(6,444-7,247)$ in 1990 to $19,723(18,941-20,518)$ in 2006 (Fig. 2). The proportion of sabbaticals to breeders did not show any temporal trend but substantial fluctuations occurred during the study period, ranging from 0.012 $(0.001-0.036)$ to $0.868(0.585-1.133)$ (Figs. 2 and $3 g)$. The proportion of sabbaticals to breeders was high $(>0.7)$ during some consecutive years (1989-1991), and mean sabbatical rate for the study period was $0.125(0.055-0.217)$. The proportion of not-yet recruited individuals relative to breeders ranged from $0.040(0.030-0.050)$ to $0.295(0.257-0.336)$ (Fig. 3h). The proportion of non-breeders (i.e., both not recruited and sabbaticals) ranged from $0.073(0.037-0.141)$ to $1.063(0.768-1.340)$ (Fig. 3i). A high proportion of nonbreeders (>0.9) occurred during 1989-1991.

Mean survival probability was lowest in one- and twoyear-old individuals, highest at three years of age and intermediate in four-year-old individuals and five-year-old or older individuals (Table 1, Fig. 3b-e). Temporal random variation in survival was highest in the youngest part of the population (one- and two-year-old), intermediate in threeyear-old, and lower in older individuals. Probability of first reproduction was highest at four years of age, lowest for older individuals, and intermediate at three years of age. The probability to skip reproduction was on average low with substantial temporal random variability (Table 1; Table S1 in Appendix S2). Fertility $\left(b_{0}\right)$ was, on average, $0.470(0.377-0.580)$ fledglings per breeding pair and year, also with strong temporal random standard deviation (Table 1, Fig. 3f).

Average detection probability increased with age and was larger for trap aware (AW) than unaware (UN) individuals (Table 1).

\section{Effect of vital rates on population growth rate}

Survival of individuals one and two years old was the main driver of the population growth rate during the study period

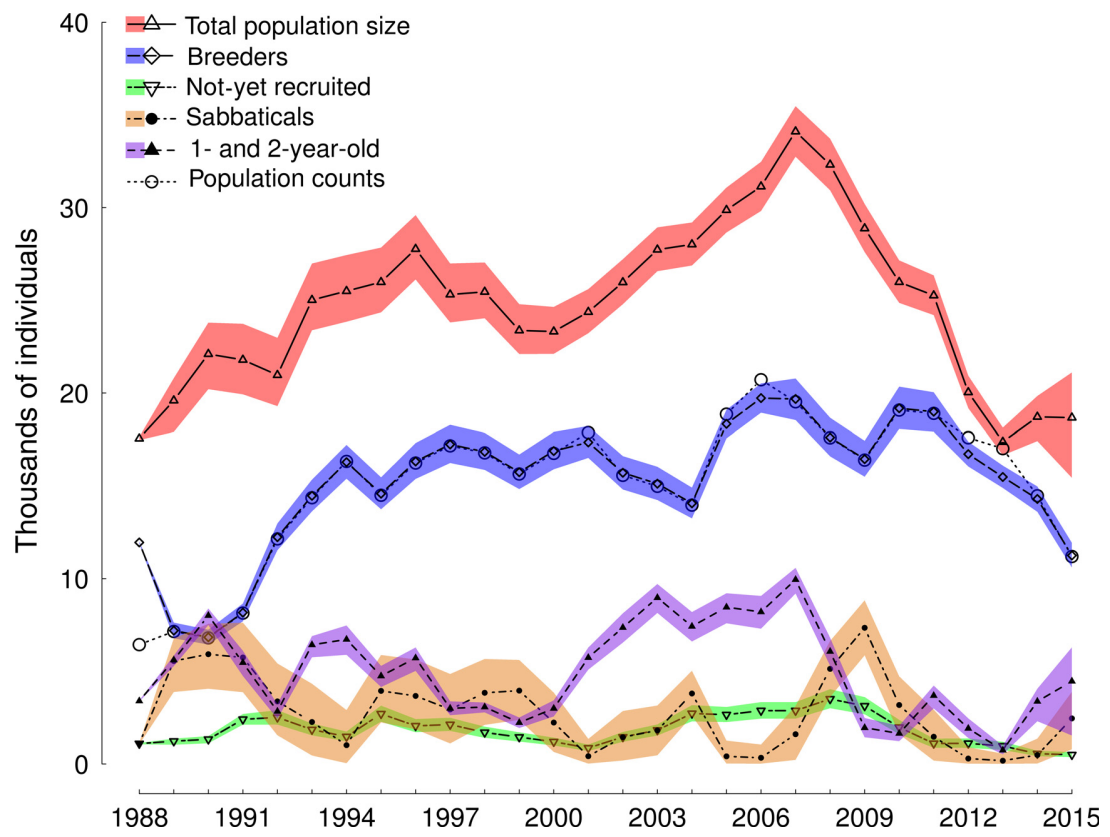

FIG. 2. Estimated and observed year-specific number of individuals of each fraction for the Audouin's Gull global population. The colored shaded areas represent the $95 \%$ credible intervals for the estimates. 
a

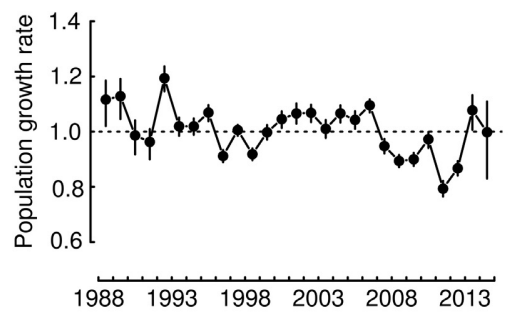

d
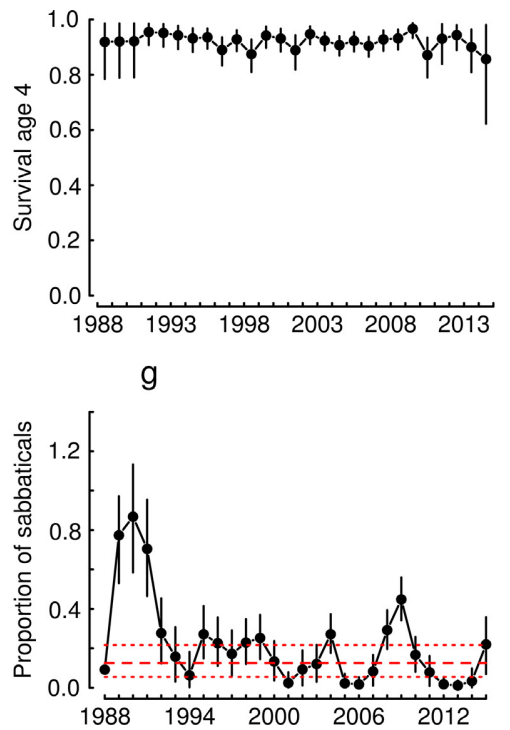

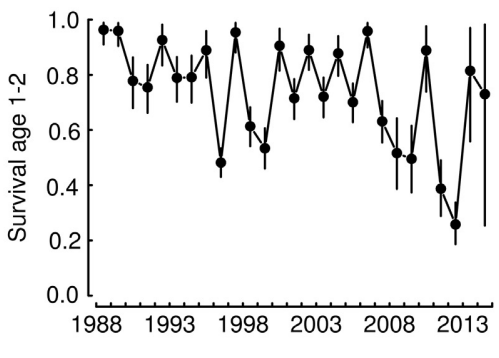

e
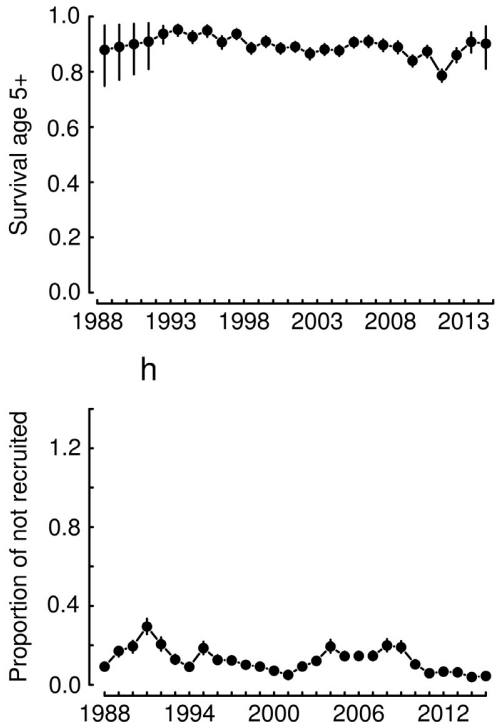

C

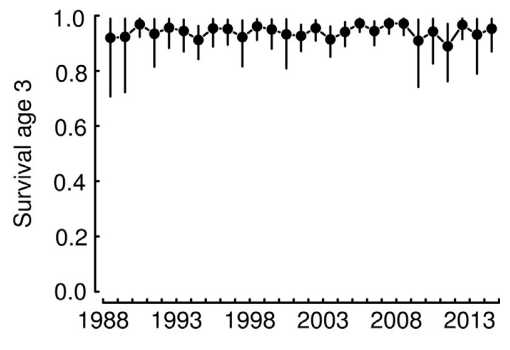

f
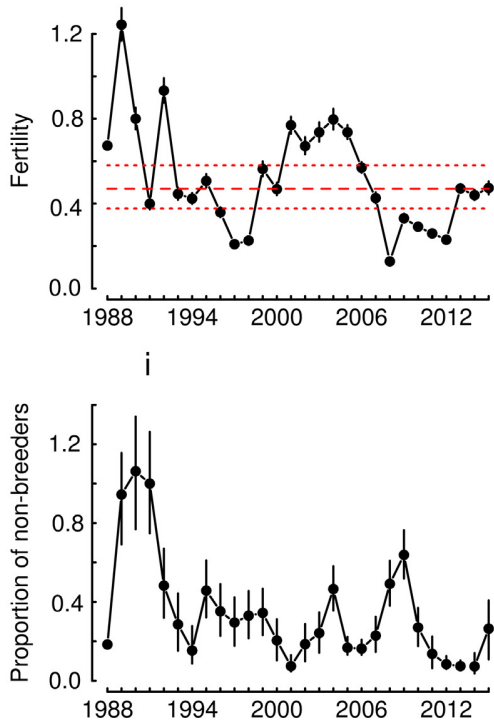

FIG. 3. (a) Estimates of population growth rate $\lambda_{t}$, (b-f) year-specific demographic rates, and (g-i) the proportion of sabbaticals, not-yet recruited individuals, and non-breeders (i.e., sabbaticals and not-yet recruited) to breeders of the Audouin's Gull population. In panels $\mathrm{f}$ and $\mathrm{g}$, the red dashed line indicates the mean from the random effects model, and the red dotted lines indicate the $95 \%$ credible intervals of the mean (not reported for the derived parameters). The vertical lines indicate the $95 \%$ credible intervals for the annual estimates. The horizontal black dotted line in panel a indicates population stability.

as suggested by the strongest correlation between this parameter and the population growth rate $\left(r^{2}=0.83,0.77-0.87\right.$; Fig. 4; Table S2 in Appendix S2). The second vital rate most influencing population growth rate was fertility $\left(r^{2}=0.73\right.$, 0.63-0.79) (Fig. 4; Table S2 in Appendix S2). Survival of individuals older than four years was also positively correlated with population growth rate at a lesser extent $\left(r^{2}=0.55,0.35-0.70\right)$ (Fig. 4; Table S2 in Appendix S2), whereas correlation with three- and four-year-old survival was close to zero (Table S2 in Appendix S2).

\section{Density dependence}

We found strong evidence of density dependence. Population growth rate $\left(\lambda_{t}\right)$ was negatively correlated with total population size, number of breeders, number of sabbaticals, and number of non-breeders at year $t$, being the number of breeders the fraction of the population showing the strongest density dependence $\left(r^{2}=-0.34,-0.43\right.$ to 0.24$)$ (Fig. 4; Table S3 in Appendix S2). Conversely, population growth rate was positively correlated with the number of one-yearold individuals $\left(r^{2}=0.16,0.07-0.26\right)$ and three-year-old individuals $\left(r^{2}=0.10,0.00-0.23\right) \quad$ (Fig. 4; Table S3 in Appendix S2). Virtually, no density dependence effect was found due to the number of two-year-old individuals or the number of not-yet recruited individuals (Table S3 in Appendix S2).

\section{DisCUSSION}

Based on long-term monitoring and information gathered on a large geographic scale, along with the use of integrated population modeling, we were able to identify the main demographic drivers of population dynamics in a long-lived species and the role of density dependence in all fractions of the population (both observable and unobservable). Our approach allowed us to assess the global species conservation status after the recent collapse of the main breeding site.

In our case study, the unobservable fraction of the population is the non-breeders. We included these non-breeders in our integrated models and, in agreement with a previous theoretical study (Lee et al. 2017), we show that these individuals play a significant role in population dynamics. We also found that the proportion of non-breeders was highly variable over the study period. The ratio of non-breeders to breeders acts as a proxy for population health and stability in some species and populations (Hunt 1998) with a rapid decrease in the number of non-breeders in seabird colonies 
TABle 1. Posterior estimates (mean, standard deviation, and percentiles) for the demographic parameters of the Audouin's Gull population.

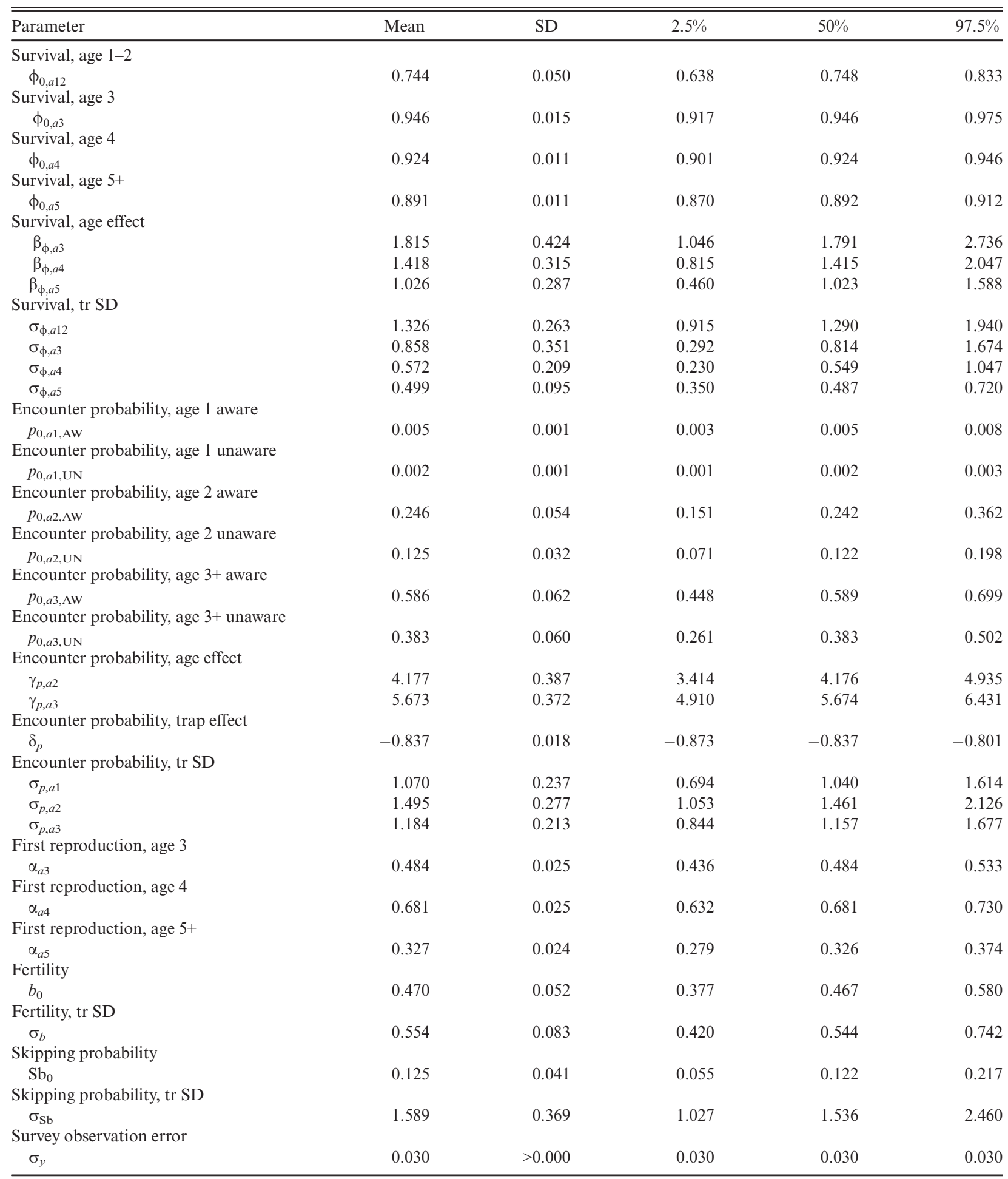

Notes: Parameters are denoted as follows: $\phi_{0, a 12}, \phi_{0, a 3}, \phi_{0, a 4}, \phi_{0, a 5}$, intercept of age-specific survival probabilities on the probability scale (" $a 12$ " one- and two-year-old, " $a 3$ " three-year-old, " $a 4$ " four-year-old, " $a 5$ " five-year-old or older); $\beta_{\phi, a 3}, \beta_{\phi, a 4}, \beta_{\phi, a 5}$, age effect on survival probability (logit scale); $\sigma_{\phi, a 12}, \sigma_{\phi, a 3}, \sigma_{\phi, a 4}, \sigma_{\phi, a 5}$, age-specific temporal random standard deviations of survival (logit scale); $p_{0, a 1, \mathrm{AW},} p_{0, a 1}$, UN, $p_{0, a 2, \mathrm{AW}}, p_{0, a 2, \mathrm{UN}}, p_{0, a 3, \mathrm{AW}}, p_{0, a 3, \mathrm{UN}}$, intercept of age-specific encounter probabilities for trap aware (AW) and unaware (UN) individuals (probability scale) (" $a 1$ " one-year-old, " $a 2$ " two-year-old, " $a 3$ " three-year-old or older); $\gamma_{\mathrm{p}, \mathrm{a} 2}, \gamma_{\mathrm{p}, \mathrm{a} 3}$, age effect on encounter probability (logit scale); $\delta_{p}$, trap response (logit scale); $\sigma_{p, a 1}, \sigma_{p, a 2}, \sigma_{p, a 3}$, age-specific temporal random standard deviations of encounter probability (logit scale); $\alpha_{a 3}, \alpha_{a 4}, \alpha_{a 5}$, age-specific probability of first reproduction; $b_{0}$, intercept of fertility (natural scale, $b_{0}=\exp \left(\mu_{b}\right)$ ); $\sigma_{b}$, fertility temporal random standard deviation (log scale); $\mathrm{Sb}_{0}$, intercept of probability to skip reproduction (probability scale); $\sigma_{\mathrm{Sb}}$, temporal random standard deviation of the probability to skip reproduction (logit scale); $\sigma_{y}$, temporal random standard deviation of the detection probability for the breeding pairs survey (log scale). Temporal random SD is abbreviated as "tr SD." 
a

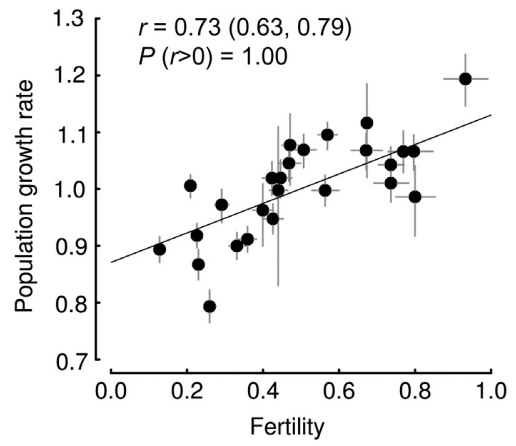

d

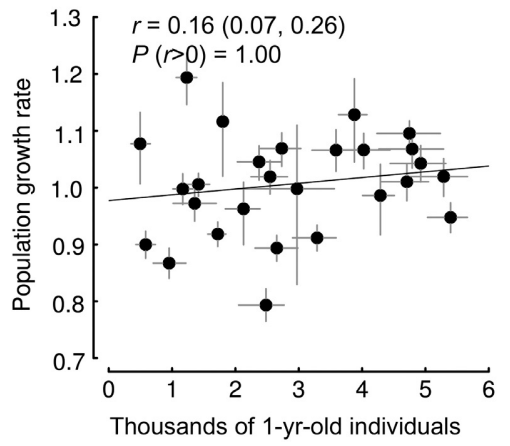

g

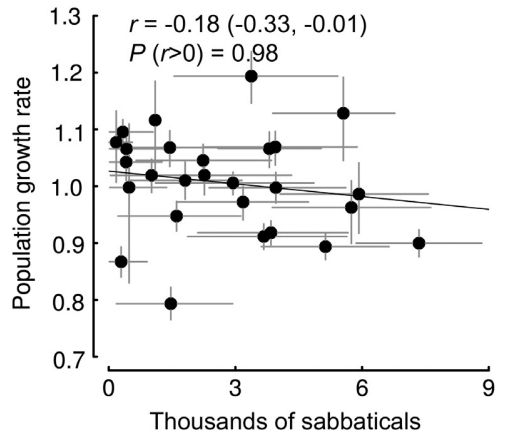

b

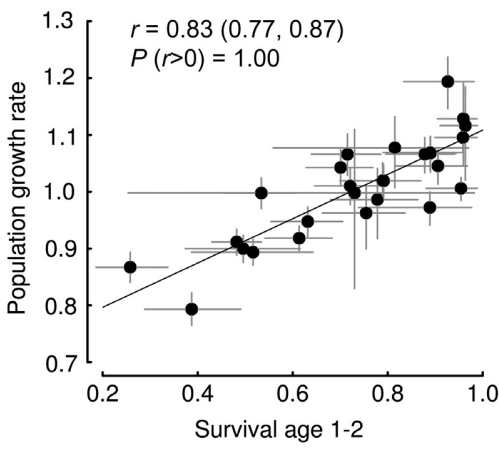

e

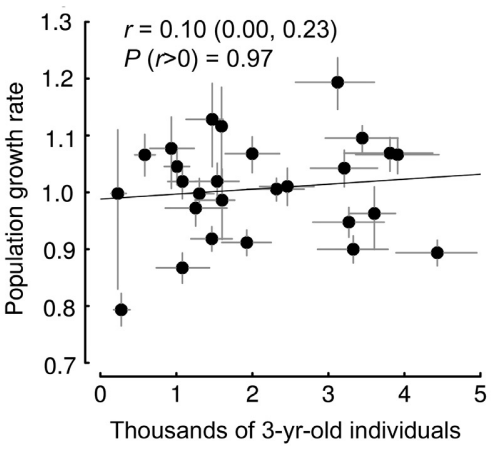

$\mathrm{h}$

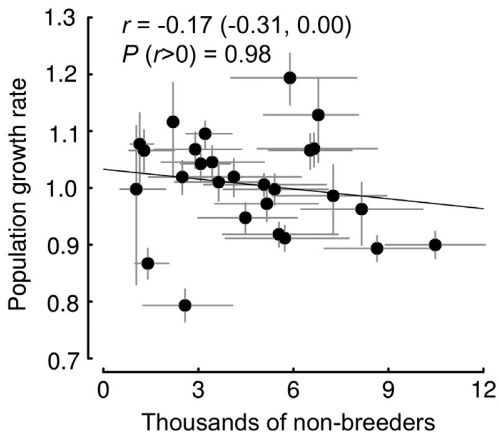

C

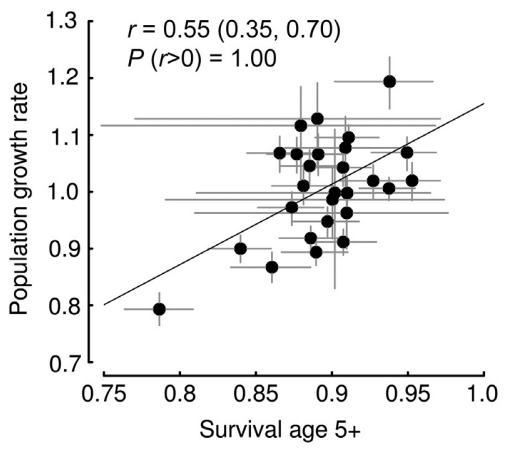

$\mathrm{f}$

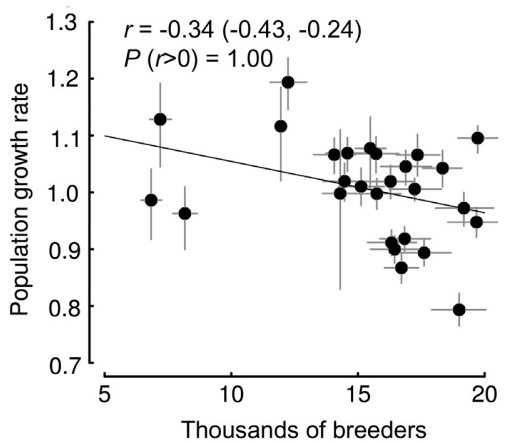

i

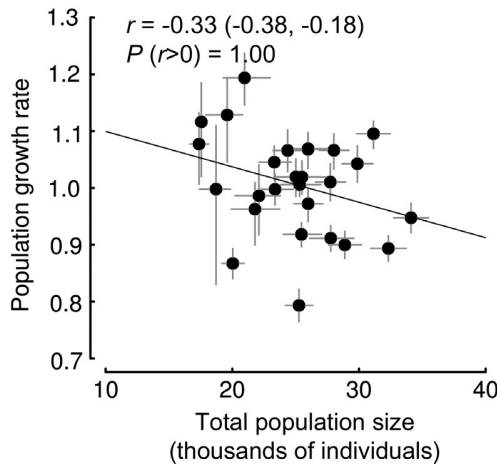

FIG. 4. Correlation between population growth rate at year $t$ and demographic variables at year $t$, where the probability of a positive or negative relationship is $\geq 0.95$. Demographic variables are (a) survival of one- and two-year-old individuals, (b) survival of five-year-old or older individuals, (c) fertility, (d) total population size, (e) number of one-year-old individuals, (f) number of three-year-old individuals, (g) number of breeders, (h) number of sabbaticals, and (i) number of non-breeders. Values reported are the probability that the estimated correlation coefficient $(r)$ is positive $(P[r>0])$ or negative $(P[r<0])$.

providing an early indication of environmental stress (Porter and Coulson 1987, Klomp and Furness 1992). In some cases, the decrease in non-breeding individuals is reflective of the buffering role of this segment of the population during harsh environmental conditions (e.g., increase of additive mortality, changes in food availability). In our case study, there is no clear indication that the numbers of nonbreeders precede significant population decline. However, we analyzed a time period that elapsed the exponential growth phase of the species and we would not expect to observe a relationship between non-breeders and population size at this stage of population dynamics. Due to their buffering role, the number of non-breeders may also be helpful to assess the potential resilience of a local population
(Durell and Clarke 2004, Penteriani et al. 2011). Based on the estimated number of Audouin's Gull non-breeders, it seems that this resilience is actually low. Recruitment is another buffering process usually observed in natural populations (Gaillard et al. 1998, Votier et al. 2008). We should acknowledge that recruitment here is assumed to be an age constant function over the study period. The lack of suitable data made it impossible to jointly analyze time variation in both the recruitment and sabbatical processes. Further analyses, perhaps focusing on just one data-rich colony, could shed some light on the role of temporal variation of recruitment in population dynamics.

We were able to estimate the role of density dependence in the global population dynamics of a long-lived species. 
Integrated population modeling allowed us to also estimate the strength of density dependence on the non-breeders even though they are unobservable. Following analyses of a general framework of population projection models that explicitly incorporated various types of non-breeders, Lee et al. (2017) suggested that further work assessing the influence of non-breeders on density-dependent dynamics is necessary. We found that the number of breeders was the fraction of the population with a stronger effect on population growth rate, but we also detected a non-negligible density dependence by individuals taking sabbaticals. This suggests that this fraction of the population is competing for food resources with breeders and other species within the same ecological guild. We think that competition may be occurring during the reproductive season, and most individuals of a species performing distant migration would skip breeding once they return to the breeding areas (Bécares et al. 2016).

When evaluating the role of the vital rates on population growth rate, we observe that the immature survival rate contributes the most to the variability in population growth during the $28 \mathrm{yr}$ of study. This is followed by the effects on fertility. Even if population growth rate in long-lived species is most sensitive to variations in adult survival, adult survival in these species usually shows less temporal variation (Pfister 1998, Sæther and Bakke 2000, Morris et al. 2011). Our work suggests that in long-lived species population, dynamics can be effectively driven by other demographic parameters such as immature survival rates or fertility. This may suddenly change if adult survival is effected, for instance by additive mortality from anthropogenic causes (e.g., fisheries' bycatch, overharvesting, electrocution in power lines). We suggest that the significant role of adult survival driving the population growth rate of the Audouin's Gulls is mainly caused by temporal variation in its bycatch mortality (Genovart et al. 2017). In the absence of this source of anthropogenic mortality, the role of adult survival on population growth rate would be even lower than the actual reported. Our integrated model points out that skipping breeding would be a bet-hedging strategy for long-lived organisms that have many reproductive chances during their lifespan and would be a part of the survival-reproduction evolutionary trade-off. In longlived species, this would prevent jeopardizing survival when environmental breeding conditions are harsh (Stearns 1992).

The main ecological drivers explaining the temporal variability of demographic parameters in this species have been previously identified. Variations in adult survival seem to be partially explained by bycatch mortality (Genovart et al. 2017) and occasional local predation by carnivores (PayoPayo et al. 2018). Other local factors, including food availability or predators, may affect other fitness parameters such as fertility (Oro et al. 1999). Great differences in the ecological drivers at a local scale may occur, and it would be interesting in the near future to quantify their relative importance acting through different demographic rates (Horswill et al. 2016).

The Audouin's Gull global population shows more than a $5 \%$ annual decrease in population during the last 10 years. Thus, from a conservation point of view, we propose an update of its conservation status. The main actual threat for the species is likely fisheries bycatch (Genovart et al. 2017), carnivore predation within some colonies (Payo-Payo et al. 2018), a general decrease of fertility due to regime shifts in small pelagic dynamics (Van Beveren et al. 2016), increased niche competition and loss of available suitable breeding habitat (Oro et al. 2009, Almaraz and Oro 2011). The species seems to respond to local disturbances with the colonization of new habitats (Payo-Payo et al. 2017); however, this process does not compensate for the global population decline. Attention should be paid to some specific regions that have remained relatively unexplored, such as northern Africa, and a tiny population in southern Portugal that has experienced marked growth in recent years (Calado et al. 2017).

We highlight the importance of long-term monitoring studies at large spatial scales for identifying the demographic parameters that have the most influence on population dynamics. Taking into account all fractions of the population, including those that are unobservable, and embracing a representative range of the population will increase our ecological understanding. This is especially true for highly mobile species and in instances when heterogeneity in local dynamics occurs. Our study improves our understanding of animal population dynamics. We provide evidence for the existence of density dependence, the important role of non-breeders in some species' population dynamics and demonstrate that in the absence of significant anthropogenic mortality, population dynamics in long-lived species may be governed by other demographic parameters with lower sensitivities than adult survival.

\section{ACKNOWLEDGMENTS}

M. Genovart and S. Tenan equally contributed to this work. This work could not have been possible without the inestimable and enormous effort made by all people involved in monitoring Audouin's Gull colonies over the decades. We would like to specially thank the staff from the Ebro Delta NP, Albert Bertolero, Toni Curcó, Julia Piccardo, Pepe Greño, Isa Afán, Jordi Muntaner, Juan Jimenez, and all the people who provided data on the species. We also thank the Regional Governments of Catalonia, Valencia, Murcia and Balearic Islands, as well as OAPN (Spanish Government) for permits to access the study sites and for logistical support. Elizabeth Rochon helped editing the English. M. Genovart is supported by a postdoctoral contract co-funded by the Regional Government of the Balearic Islands and the European Social Fund. Funds were partially provided by the Spanish Ministry of Economy (ref. CGL2013-42203-R and CGL2017-85210-P) and the European Union (H2020 RIA- MINOUW Grant, ref. H2020- 634495). We would also thank Dr. John Sauer and two anonymous referees for providing helpful and constructive comments for improving the manuscript.

\section{Literature Cited}

Abadi, F., O. Gimenez, R. Arlettaz, and M. Schaub. 2010. An assessment of integrated population models: bias, accuracy, and violation of the assumption of independence. Ecology 91:7-14.

Abadi, F., O. Gimenez, H. Jakober, W. Stauber, R. Arlettaz, and M. Schaub. 2012. Estimating the strength of density dependence in the presence of observation errors using integrated population models. Ecological Modelling 242:1-9.

Almaraz, P., and D. Oro. 2011. Size-mediated non-trophic interactions and stochastic predation drive assembly and dynamics in a seabird community. Ecology 92:1948-1958.

Bailey, L. L., W. L. Kendall, D. R. Church, and H. M. Wilbur. 2004. Estimating survival and breeding probability for pond-breeding amphibians: a modified robust design. Ecology 85:2456-2466.

Bécares, J., J. Arcos, and D. Oro. 2016. Migración y ecología espacial de la gaviota de Audouin en el Mediterráneo occidental y noroeste africano. Monografía $\mathrm{n}^{\circ} 1$ del programa Migra. SEO/BirdLife, Madrid, Spain. 
Besbeas, P., S. N. Freeman, B. J. T. Morgan, and E. A. Catchpole. 2002. Integrating mark-recapture-recovery and census data to estimate animal abundance and demographic parameters. Biometrics 58:540-547.

BirdLife International. 2017. Larus audouinii (amended version of 2016 assessment). The IUCN Red List of Threatened Species 2017: e.T22694313A110634317. http://dx.doi.org/10.2305/IUCN UK.2017-1.RLTS.T22694313A110634317.en

Bjørkvoll, E., A. M. Lee, V. Grøtan, B.-E. Sæther, A. Stien, S Engen, S. Albon, L. E. Loe, and B. B. Hansen. 2016. Demographic buffering of life histories? Implications of the choice of measurement scale. Ecology 97:40-47.

Brooks, S. P., and A. Gelman. 1998. General methods for monitoring convergence of iterative simulations. Journal of Computational and Graphical Statistics 7:434-455.

Calado, J. G., D. M. Matos, J. A. Ramos, F. Moniz, F. R. Ceia, J. P. Granadeiro, and V. H. Paiva. 2017. Seasonal and annual differences in the foraging ecology of two gull species breeding in sympatry and their use of fishery discards. Journal of Avian Biology 49: https://doi.org/10.1111/jav.01463

Cam, E., J. E. Hines, J.-Y. Monnat, J. D. Nichols, and E. Danchin. 1998. Are adult non-breeders prudent parents? The Kittiwake model. Ecology 79:2917-2930.

Caswell, H. 2000. Prospective and retrospective perturbation analyses: their roles in conservation biology. Ecology 81:619-627.

Clobert, J., J.-F. Le Galliard, J. Cote, S. Meylan, and M. Massot. 2009. Informed dispersal, heterogeneity in animal dispersal syndromes and the dynamics of spatially structured populations. Ecology Letters 12:197-209.

Crouse, D. T., L. B. Crowder, and H. Caswell. 1987. A stage-based population model for loggerhead sea turtles and implications for conservation. Ecology 68:1412-1423.

Dennis, B., J. M. Ponciano, S. R. Lele, M. L. Taper, and D. F. Staples. 2006. Estimating density dependence, process noise, and observation error. Ecological Monographs 76:323-341.

Durell, S., and R. T. Clarke. 2004. The buffer effect of non-breeding birds and the timing of farmland bird declines. Biological Conservation 120:375-382.

Fernández-Chacón, A., M. Genovart, R. Pradel, G. Tavecchia, A. Bertolero, J. Piccardo, M. G. Forero, I. Afán, J. Muntaner, and D. Oro. 2013. When to stay, when to disperse and where to go: survival and dispersal patterns in a spatially structured seabird population. Ecography 36:1117-1126.

Freckleton, R. P., A. R. Watkinson, R. E. Green, and W. J. Sutherland. 2006. Census error and the detection of density dependence. Journal of Animal Ecology 75:837-851.

Frederiksen, M., M. P. Harris, and S. Wanless. 2005. Inter-population variation in demographic parameters: a neglected subject? Oikos 111:209-214.

Gaillard, J.-M., and N. G. Yoccoz. 2003. Temporal variation in survival of mammals: A case of environmental canalization? Ecology 84:3294-3306.

Gaillard, J.-M., M. Festa-Bianchet, and N. G. Yoccoz. 1998. Population dynamics of large herbivores: variable recruitment with constant adult survival. Trends in Ecology \& Evolution 13:58-63.

Ganzhorn, J. U., and J. Schmid. 1998. Different population dynamics of Microcebus murinus in primary and secondary deciduous dry forests of Madagascar. International Journal of Primatology 19:785-796.

Genovart, M., L. Jover, X. Ruiz, and D. Oro. 2003. Offspring sex ratios in subcolonies of Audouin's gull, Larus audouinii, with differential breeding performance. Canadian Journal of Zoology 81:905-910.

Genovart, M., R. Pradel, and D. Oro. 2012. Exploiting uncertain ecological fieldwork data with multi-event capture-recapture modelling: an example with bird sex assignment. Journal of Animal Ecology 81:970-977.

Genovart, M., D. F. Doak, J.-M. Igual, S. Sponza, J. Kralj, and D. Oro. 2017. Varying demographic impacts of different fisheries on three Mediterranean seabird species. Global Change Biology 23:3012-3029.

Genovart, M., J. Bécares, J.-M. Igual, A. Martínez-Abraín, R. Escandell, A. Sánchez, B. Rodríguez, J. M. Arcos, and D. Oro. 2018. Differential adult survival at close seabird colonies: The importance of spatial foraging segregation and bycatch risk during the breeding season. Global Change Biology 24:1279-1290.

Grosbois, V., O. Gimenez, J.-M. Gaillard, R. Pradel, C. Barbraud, J. Clobert, A. P. Møller, and H. Weimerskirch. 2008. Assessing the impact of climate variation on survival in vertebrate populations. Biological Reviews 83:357-399.

Grosbois, V., M. P. Harris, T. Anker-Nilssen, R. H. McCleery, D. N. Shaw, B. J. T. Morgan, and O. Gimenez. 2009. Modeling survival at multi-population scales using mark-recapture data. Ecology 90:2922-2932.

Hanski, I., P. Foley, and M. Hassell. 1996. Random walks in a metapopulation: How much density dependence is necessary for long-term persistence? Journal of Animal Ecology 65:274-282.

Horswill, C., N. Ratcliffe, J. A. Green, R. A. Phillips, P. N. Trathan, and J. Matthiopoulos. 2016. Unravelling the relative roles of topdown and bottom-up forces driving population change in an oceanic predator. Ecology 97:1919-1928.

Hunt, W. G. 1998. Raptor floaters at Moffat's equilibrium. Oikos 82:191-197.

Kery, M., and M. Schaub. 2011. Bayesian population analysis using WinBUGS: A hierarchical perspective. Academic Press, Waltham, Massachussets, USA.

Klomp, N. I., and R. W. Furness. 1992. Non-breeders as a buffer against environmental stress: declines in numbers of Great Skuas on Foula, Shetland, and prediction of future recruitment. Journal of Applied Ecology 29:341-348.

Knape, J. 2008. Estimability of density dependence in models of time series data. Ecology 89:2994-3000.

Lande, R., S. Engen, and B.-E. Sæther. 2003. Stochastic population dynamics in ecology and conservation. Oxford University Press, Oxford, UK.

Lebreton, J.-D., and O. Gimenez. 2013. Detecting and estimating density dependence in wildlife populations. Journal of Wildlife Management 77:12-23.

Lebreton, J.-D., K. P. Burnham, J. Clobert, and D. R. Anderson. 1992. Modeling survival and testing biological hypotheses using marked animals: a unified approach with case studies. Ecological Monographs 62:67-118.

Lee, A. M., J. M. Reid, and S. R. Beissinger. 2017. Modelling effects of nonbreeders on population growth estimates. Journal of Animal Ecology 86:75-87.

Morris, W. F., and D. F. Doak. 2004. Buffering of life-histories against environmental stochasticity: accounting for a spurious correlation between the variabilities of vital rates and their contributions to fitness. American Naturalist 165:579-590.

Morris, W. F., J. Altmann, D. K. Brockman, M. Cords, L. M. Fedigan, A. E. Pusey, T. S. Stoinski, A. M. Bronikowski, S. C. Alberts, and K. B. Strier. 2011. Low demographic variability in wild primate populations: fitness impacts of variation, covariation, and serial correlation in vital rates. American Naturalist 177:E14 E28.

Muths, E., R. D. Scherer, and B. A. Lambert. 2010. Unbiased survival estimates and evidence for skipped breeding opportunities in females. Methods in Ecology and Evolution 1:123-130.

Nichols, J. D. 1992. Capture-recapture models. BioScience 42:94 102.

Oro, D., and R. Pradel. 2000. Determinants of local recruitment in a growing colony of Audouin's gull. Journal of Animal Ecology 69:119-132.

Oro, D., and G. D. Ruxton. 2001. The formation and growth of seabird colonies: Audouin's gull as a case study. Journal of Animal Ecology 70:527-535.

Oro, D., R. Pradel, and J.-D. Lebreton. 1999. Food availability and nest predation influence life history traits in Audouin's gull, Larus audouinii. Oecologia 118:438-445. 
Oro, D., A. Pérez-Rodríguez, A. Martínez-Vilalta, A. Bertolero, F. Vidal, and M. Genovart. 2009. Interference competition in a threatened seabird community: A paradox for a successful conservation. Biological Conservation 142:1830-1835.

Oro, D., G. Tavecchia, and M. Genovart. 2010. Comparing demographic parameters for philopatric and immigrant individuals in a long-lived bird adapted to unstable habitats. Oecologia 165:935945.

Pardo, D., H. Weimerskirch, and C. Barbraud. 2013. When celibacy matters: incorporating non-breeders improves demographic parameter estimates. PLoS ONE 8:e60389.

Payo-Payo, A., M. Genovart, A. Bertolero, R. Pradel, and D. Oro. 2016. Consecutive cohort effects driven by density-dependence and climate influence early-life survival in a long-lived bird. Proceedings of the Royal Society B 283:20153042.

Payo-Payo, A., M. Genovart, A. Sanz-Aguilar, J. L. Greño, M. García-Tarrasón, A. Bertolero, J. Piccardo, and D. Oro. 2017. Colonisation in social species: the importance of breeding experience for dispersal in overcoming information barriers. Scientific Reports 7:42866.

Payo-Payo, A., A. Sanz-Aguilar, M. Genovart, A. Bertolero, J. Piccardo, D. Camps, J. Ruiz-Olmo, and D. Oro. 2018. Predator arrival elicits differential dispersal, change in age structure and reproductive performance in a prey population. Scientific Reports $8: 1971$.

Penteriani, V., M. Ferrer, and M. M. Delgado. 2011. Floater strategies and dynamics in birds, and their importance in conservation biology: towards an understanding of nonbreeders in avian populations. Animal Conservation 14:233-241.

Pfister, C. A. 1998. Patterns of variance in stage-structured populations: Evolutionary predictions and ecological implications. Proceedings of the National Academy of Sciences USA 95:213-218.

Plummer, M 2003. JAGS: a program for analysis of Bayesian graphical models using Gibbs sampling. Pages 125. Proceedings of the 3 rd international workshop on distributed statistical computing. Technische Universit at Wien, Wien, Austria.

Plummer, M. 2016. rjags: Bayesian graphical models using MCMC. $\mathrm{R}$ package version 4-6. https:/CRAN.R-project.org/package=rjags

Porter, J. M., and J. C. Coulson. 1987. Long-term changes in recruitment to the breeding group, and the quality of recruits at a Kittiwake Rissa tridactyla colony. Journal of Animal Ecology 56:675-689.
R Core Team. 2012. R: A language and environment for statistical computing. R Foundation for Statistical Computing, Vienna, Austria. http://www.r-project.org/

Sæther, B.-E., and O. Bakke. 2000. Avian life history variation and contribution of demographic traits to the population growth rate. Ecology 81:642-653.

Sæther, B.-E., et al. 2016. Demographic routes to variability and regulation in bird populations. Nature Communications 7:12001.

Schaub, M., and F. Abadi. 2011. Integrated population models: a novel analysis framework for deeper insights into population dynamics. Journal of Ornithology 152:227-237.

Schaub, M., H. Jakober, and W. Stauber. 2013. Strong contribution of immigration to local population regulation: evidence from a migratory passerine. Ecology 94:1828-1838.

Sibly, R. M., and J. Hone. 2002. Population growth rate and its determinants: an overview. Philosophical Transactions of the Royal Society B 357:1153-1170.

Stearns, S. C. 1992. The evolution of life histories. OUP Oxford, New York, New York, USA.

Stenseth, N. C., and W. Z. Lidicker. 1992. The study of dispersal: a conceptual guide. Pages 5-20 in N. C. Stenseth and W. Z. Lidicker, editors. Animal dispersal. Springer, Dordrecht, The Netherlands.

Tenan, S., J. Adrover, A. M. Navarro, F. Sergio, and G. Tavecchia. 2012. Demographic consequences of poison-related mortality in a threatened bird of prey. PLoS ONE 7:e49187.

Tenan, S., A. Iemma, N. Bragalanti, P. Pedrini, M. De Barba, E. Randi, C. Groff, and M. Genovart. 2016. Evaluating mortality rates with a novel integrated framework for non-monogamous species. Conservation Biology 30:1307-1319.

Tenan, S., M. Fasola, S. Volponi, and G. Tavecchia. 2017. Conspecific and not performance-based attraction on immigrants drives colony growth in a waterbird. Journal of Animal Ecology 86:1074-1081.

Van Beveren, E., J.-M. Fromentin, T. Rouyer, S. Bonhommeau, P. Brosset, and C. Saraux. 2016. The fisheries history of small pelagics in the Northern Mediterranean. ICES Journal of Marine Science 73:1474-1484.

Votier, S. C., T. R. Birkhead, D. Oro, M. Trinder, M. J. Grantham, J. A. Clark, R. H. McCleery, and B. J. Hatchwell. 2008. Recruitment and survival of immature seabirds in relation to oil spills and climate variability. Journal of Animal Ecology 77:974-983.

\section{SUPPORTING INFORMATION}

Additional supporting information may be found in the online version of this article at http://onlinelibrary.wiley.com/doi/10.1002/ecy. 2515/suppinfo

\section{DAta ACCESSIBILITy}

Data are available from DIGITAL.CSIC at http://hdl.handle.net/10261/166903 